\title{
Reasoning about Rational, but not Logically Omniscient Agents
}

\author{
Ho Ngoc DUC \\ Institute of Informatics, University of Leipzig \\ PO Box 920, D-04009 Leipzig, Germany \\ Phone: +49-341-9732283, Fax: +49-341-9739348 \\ Email: duc@informatik.uni-leipzig.de \\ http://www.informatik.uni-leipzig.de/ ${ }^{\sim}$ duc/home.html
}

\begin{abstract}
We propose in the paper a new solution to the so-called Logical Omniscience Problem of epistemic logic. Almost all attempts in the literature to solve this problem consist in weakening the standard epistemic systems: weaker systems are considered where the agents do not possess the full reasoning capacities of ideal reasoners. We shall argue that this solution is not satisfactory: in this way omniscience can be avoided, but many intuitions about the concepts of knowledge and belief get lost. We shall show that axioms for epistemic logics must have the following form: if the agent knows all premises of a valid inference rule, and if she thinks hard enough, then she will know the conclusion. To formalize such an idea, we propose to "dynamize" epistemic logic, that is, to introduce a dynamic component into the language. We develop a logic based on this idea and show that it is suitable for formalizing the notion of actual, or explicit knowledge.
\end{abstract}

Keywords: Logics of knowledge and belief, Logical omniscience, Resource bounded reasoner, Dynamic epistemic logic, Knowledge and time

\section{Introduction and Preliminaries}

Epistemic logic, or the logic of the concepts of knowledge and belief, has established as an autonomous branch of logic since the work of Hintikka ([13].) The subject has been studied extensively by philosophers, linguists, economists, and, more recently, computer scientists. ${ }^{1}$ In computer science and artificial intelligence epistemic logic has been used for analyzing distributed systems, for knowledge representation, or for the specification of multi-agent systems.

However, it is a very controversial matter whether epistemic logic is suitable for these purposes. Most systems of epistemic logic have been developed in analogy to

\footnotetext{
${ }^{1}$ For recent reviews and extensive bibliographies on the subject, see e.g., [9], [10], [24], [7].
} 
modal logic. This approach has clearly some advantages. Many results and methods of modal logic can be transferred to epistemic logic, notably the techniques of the possible worlds semantics. There are, however, severe objections against the modal approach in epistemic logic. The most serious problem of this approach is perhaps the so-called "logical omniscience problem (LOP)." It can be described informally as follows. According to the standard approach, the agent should be an ideal logician and reasoner in the following sense: she knows all logical truths, can (actually) draw all consequences of a certain sentence and can identify all logical equivalences of a given sentence. Such requirements are clearly too strong for a real agent, human or non-human. Thus, the standard systems cannot capture the notion of knowledge and belief adequately. The problem of logical omniscience is a severe obstacle for the applicability of epistemic logic. For that reason, many attempts have been undertaken to solve this problem. The goal of our paper is to assess how successful these attempts can be and then to propose another solution to the LOP.

Before going on, let us state the problem more precisely. In the paper we consider the concept of knowledge only, but the main arguments apply to the concept of belief, too. By "modal epistemic logic" we mean, as proposed by Wuttich in [25], those systems of epistemic logic which are developed in the modal tradition. Let us assume a set Agt of $n$ agents. The language of modal epistemic logic is built up from a set $A t$ of propositional letters using the usual Boolean connectives of negation and implication and the operators $K_{i}$, each for one agent $i$. In the AI community the systems $\mathrm{S} 4_{\mathrm{n}}$ and $\mathrm{S} 5_{\mathrm{n}}$ are most often considered as logics of rational knowledge for the case of $n$ agents. They consist of $n$ copies of the modal systems S4 and S5, respectively. Formally:

Definition 1 (The language of epistemic logic) Let $A t$ be a set of atomic formulae and $A g t=\{1, \ldots, n\}$ a set of agents. $L_{E}$ is the least set such that

1. $A t \subseteq L_{E}$

2. If $A \in L_{E}$ then $\neg A \in L_{E}$

3. If $A \in L_{E}$ and $B \in L_{E}$ then $(A \rightarrow B) \in L_{E}$

4. If $A \in L_{E}$ and $i \in A g t$ then $K_{i} A \in L_{E}$

The intended interpretation of the formula $K_{i} A$ is that the agent $i$ knows that $A$. The other truth-functional connectives are defined as usual. An objective formula is one that does not contain any knowledge operator. We adopt the standard conventions concerning the use of parentheses.

Definition 2 (The logics $\mathrm{S} 4_{\mathrm{n}}$ and $\mathrm{S} 5_{\mathrm{n}}$ ) The modal epistemic logic $\mathrm{S} 5_{\mathrm{n}}$ (for the case of $n$ agents) has the following axiom schemata:

PC. All theorems of the propositional calculus (PC).

K. $K_{i}(A \rightarrow B) \rightarrow\left(K_{i} A \rightarrow K_{i} B\right)$

T. $K_{i} A \rightarrow A$

4. $K_{i} A \rightarrow K_{i} K_{i} A$ 
5. $\neg K_{i} A \rightarrow K_{i} \neg K_{i} A$

The rules of inference are:

MP. Modus ponens: if $A$ and $A \rightarrow B$ are theorems then $B$ is a theorem.

NEC. Necessitation: if $A$ is a theorem then so is $K_{i} A$.

The logic $\mathrm{S} 4_{\mathrm{n}}$ is obtained by dropping the axiom schema 5 from the above axiomatization of $\mathrm{S} 5_{\mathrm{n}}$.

The notions of proof, of theoremhood etc. with respect to a system $\mathrm{S}$ of modal epistemic logic is defined as usual. The symbol $\vdash_{S} A$ is used to denote the fact that $A$ is a theorem of $\mathrm{S}$. A formula $A$ is said to be an $\mathrm{S}$-consequence from a set $X$ of formulae, denoted $X \vdash_{S} A$, just in case there are some $B_{1}, \ldots, B_{m} \in X$ such that $B_{1} \wedge \ldots \wedge B_{m} \rightarrow A$ is a theorem of $\mathrm{S}$. If the system under consideration is clear from the context, we can omit the index and write $X \vdash A$ instead.

Axiom K says that an agent's knowledge is closed under modus ponens. Axiom T states that knowledge implies truth. (It follows that an agent's knowledge is consistent.) The axioms 4 and 5 are called positive and negative introspection axioms, respectively. They say that an agent is aware of what she knows and what she does not know. It is generally accepted that negative introspection is a more demanding condition than positive introspection. Therefore many researchers argue that it is more reasonable to adopt $\mathrm{S} 4_{n}$ as the logic of knowledge. In case one needs to distinguish between knowledge and belief, one can drop the schema $\mathrm{T}$ or replace it by the weaker axiom $\mathrm{D}$, that is, the schema $K_{i} A \rightarrow \neg K_{i} \neg A$. The system obtained from $\mathrm{S} 5_{\mathrm{n}}$ by replacing the schema $\mathrm{T}$ by the schema $\mathrm{D}$ is known as KD45n and is considered by many researchers as the standard logic of rational belief. Besides these three systems sometimes other systems are also considered. The minimal normal modal system, containing $K$ as the only modal axiom, is called the system $K_{n}$, or just $K$ in the case of one agent.

It is well-known that the most common systems of modal epistemic logic can be determined by suitable classes of Kripke models with $n$ accessibility relations. In particular, the accessibility relations of $\mathrm{S} 5_{\mathrm{n}}$-models are equivalence relations, those of $\mathrm{S} 4 \mathrm{n}_{\mathrm{n}}$-models are reflexive and transitive, and KD $45_{\mathrm{n}}$-models are serial, transitive, and Euclidean (cf. [2], [8], [11], [7].)

The logical omniscience problem for modal epistemic logic can be stated as follows. The following inference rules are valid for $\mathrm{S} 5_{\mathrm{n}}$ and related systems:

NEC. If $A$ is a theorem then so is $K_{i} A$

MON. If $A \rightarrow B$ is a theorem then so is $K_{i} A \rightarrow K_{i} B$

CGR. If $A \leftrightarrow B$ is a theorem then so is $K_{i} A \leftrightarrow K_{i} B$

The three rules NEC, MON, CGR are called necessitation rule, monotony rule, and congruence rule, respectively. They are derivable when the schema $K$ and the necessitation rule are assumed, that is, in the minimal modal system $K_{n}$ already. An agent who is described by such a logic is said to be logically omniscient, because she knows all logical truths (according to NEC), she knows all logically consequences 
of a sentence that she knows (according to MON), and she can identify all logical equivalent sentences of a given sentence, according to CGR. Such an agent cannot be a real one. No human agent has such reasoning capacities. We cannot build artificial agents that possess the reasoning power described by $\mathrm{S} 5_{\mathrm{n}}$ and related systems. If we consider real agents and ask what they actually know, we can check empirically that an agent's knowledge is often not closed any logical law. Realistic agents may know a very restricted set of logical laws. They know only some, but not all logical consequences of their knowledge. We cannot realistically expect an agent's knowledge to be closed under even very elementary logical laws, e.g., modus ponens. That is, even the axiom schema $\mathrm{K}$ is a strong idealization. Modal epistemic logics do not capture the notions of knowledge and belief adequately.

If we agree that modal epistemic logics do not describe what agents actually know, we can ask the question: what do they describe then? Well, they are logics of a related, but different concept. It is remarked by several authors that the laws of these systems are much more acceptable if the formula $K_{i} A$ is read "the agent $i$ knows $A$ implicitly" ([18], [6],) " $A$ follows from $i$ 's knowledge" ([7]), "the agent $i$ carries the information $A$ " ([1],) or "the agent $i$ possibly knows $A$ ", instead of "the agent $i$ knows $A$ ". Modal epistemic logics should be interpreted as logics of possible, or implicit knowledge, and not as logics of actual, or explicit knowledge.

For reasoning about agents the concept of actual knowledge is much more important than that of possible knowledge. In order to predict or to explain an agent's actions we need to know what the agent actually knows, and not what she possibly knows. For modeling realistic agents we need other logics which are capable of capturing the concept of actual knowledge. Such logic may not suffer from the LOP. In the next section we shall discuss some common ways to solve this problem. After showing that the strategy of weakening epistemic logic has many disadvantages we shall propose an alternative approach to the problem. The intuitions of our strategy will be explained in section 3 . A formal system which can cope with the problems of the traditional approaches will be developed in section 4 following the new strategy. The paper closes with a discussion of related works, open problems and directions for future work.

\section{Strategies to Avoid Logical Omniscience}

An obvious strategy to solve the logical omniscience problem is to weaken epistemic logic. One denies the universal validity of the mentioned inference rules NEC, MON, and CGR, or one of the essential axioms like K. In fact, almost all attempts to solve the LOP have in common that they consider systems that are weaker than the standard modal epistemic logics (cf. [3], [5], [6], [14], [18], [21], [22], [23], [25].) One can construct systems that falsify either the inference rules or the axioms of the standard modal systems. For example, modal systems which are not normal can be used to describe an agent who does not know all logical truths. If we use neighborhood semantics instead of Kripke semantics, we can get weaker modal systems (the socalled classical systems) for which neither the rule NEC nor the axiom schema K is valid, therefore the agents' knowledge is not closed under logical consequence (cf. [2], [22].) In this way, the original version of the LOP could be solved. But here some care is needed: some systems solve the original version, but not other versions of the 
LOP. If the monotony rule and/or the congruence rule are valid for a system, or if an agent does not know all theorems of classical logic, but knows all theorem of another (nontrivial) logic, then the agent in this system must still be viewed as ideal: real agents simply never achieve such reasoning capacities which can be described by such a system. For example, the agent described by the minimal classical modal system E (cf. [22], [2]) knows all logical equivalences of a sentence she knows. An agent in Levesque's logic ([18]) does not know all truths of classical logic, but she knows all theorems of a relevance logic. In the same way, an agent in Ho Ngoc Duc's system ([3]) does not know all classical theorems, but knows all theorems of a three-valued logic. Such attempts cannot be considered satisfactory solutions to the LOP.

A number of systems have been proposed which assume still more restricted reasoning capacities of the agents. To construct such a system we can postulate, for example, that the agent only knows some "obvious" logical truths, but not necessarily the "more complicated" ones. We can assume that the agent can draw all "obvious" consequences, but not any arbitrary consequence of a certain sentence. We can do it by postulating that the deduction mechanism of the agents is not complete, that is, it is not powerful enough to allow the agents to draw all logical consequences of their knowledge. The regularities of an agent's knowledge could be formalized by a set of suitable axioms. The more axioms are postulated, the more rational is the agent. With the aid of weak epistemic logics we can classify the agents according to their logical capacities. This approach is pursued by Stelzner in his "parameterized epistemic logic" ([23],) or Konolige in his "deduction model" ([16].) If the agent's inference mechanism is kept very weak, then logical omniscience could be avoided. Besides this axiomatic approach we can also pursue a more semantical approach. One can show that logical omniscience can be avoided if one allow "impossible possible worlds" in which the valuation of the sentences of the language is arbitrary. In other words, the logical laws do not hold in the "impossible possible worlds" ([22], [21]). Another solution is to introduce a new operator of awareness into the language and to require that belief include awareness ([6].) Because it is possible that the agent is aware of some sentence but she is not aware of its logical consequences or its equivalent sentences, the theorems and inference rules of modal epistemic systems do not hold in general.

Although the deduction model, the approach with impossible possible worlds and the approach with the awareness operator solve the LOP technically, they cannot be regarded satisfactory. New problems arise in these approaches besides the old problems of the possible worlds approach. Here we shall not discuss these problems in details, nor shall we try to improve any of these approaches. We shall rather present a more fundamental criticism of the common strategy of all these approaches, namely the strategy of weakening epistemic logic.

The discussion of the logical omniscience problem in the literature has concentrated mainly on the issue: in which way can logical omniscience be avoided. But the LOP has another aspect which is often overlooked in the discussion: what is left if one restricts the reasoning capacities of the agents, for example by denying the validity of the rules NEC, MON and CGR or of the axiom schema K? Is there still a reasonable way to describe an agent's knowledge if the regularities of the agent's knowledge is too weak to be described by these axioms and rules?

An attempt to cope with this challenge is to postulate axioms which describe the 
regularities of the agent's knowledge. Such axioms shall express the intuitive idea that the agent is somehow rational, or logical. The axioms are generally of the form: the belief set of the agent is closed under a certain valid rule of inference of logic, i.e., if all premises of the rule are known, then the conclusion is known. (This is also the general form of a theorem of a standard epistemic logic.) In this way we can get subsystems of the logic K which do not suffer from the LOP. The strategy of weakening epistemic logic allows us to consider agents who are very restricted in their reasoning capacities. We can describe and classify them according to their rationality. We would have a hierarchy of agents: some agents are ideal, who are modeled by the logic K or its extensions, others are less ideal and can be described by its subsystems.

The strategy of weakening epistemic logic solves the logical omniscience problem. However, the disadvantages cannot be overlooked. First, this approach is only suited to analyze static knowledge, that is, we can at most describe the knowledge sets at one single time point. This is of course not a deficiency of these logics alone, but of most epistemic logics developed up to now. Second, the categories of agents we describe and classify by our logics are merely imaginary: they do not exist in reality. It is very implausible to assume that there are agents who always think in some fixed patterns which can be captured by one of our logics. Each agent represents rather some mixture of several logics, at some time point they can be described by one, at other time points by another, and still at other by none of our logics at all. Third, however weak our postulates may be, they may still be too strong for some agents. Given the information that an agent's knowledge includes a set $X$ of sentences, in reality we can never infer reliably that the agent knows all sentences of the deductive closure $c l_{S}(X)$ of $X$ with respect to a deductive system $S$, even if we suppose $S$ to be very weak (but not degenerate in the sense that $c l_{S}(X)=X$.) This point has led many people to raise the question if epistemic logic is possible at all, or do we have to leave the realm of logic when reasoning about knowledge and belief ([15], [1].) Fourth, we have the feeling that our logics are too weak. Surely, we want to avoid logical omniscience. On the other hand, we are interested in having epistemic logics which are strong enough to allow sufficiently many conclusions from a given set of facts we know about the agent's propositional attitudes. We want to have agents who do know at least a (sufficiently) large class of logical truths, and can draw sufficiently many conclusions from their knowledge. This is the dilemma on logical omniscience on the one side and logical ignorance on the other side. That is why we ask the question before: what is left from epistemic logic if we deny the validity of the axiom $\mathrm{K}$ or of the congruence rule and stronger principles? What we need is something between two extremes. Can we have some reasonable thing like that?

Our goal is to show that we can solve this dilemma. We shall now propose another strategy to solve the logical omniscience problem which also solves the problem of logical ignorance. Our strategy starts with the observation that the laws of (classical) logic are not sentences about the world, they do not tell us anything about what is the case in the world. If we say that the epistemic agent knows the laws of logic, we do not mean that she knows some facts about the world, but rather that she is able to use these laws to draw conclusions from what she already knows. The laws of logic are what the agent knows implicitly; she does not need to possess them permanently. It suffices if she can recall them when she needs them in order to infer new information from her explicit data base. At a given time the set of logical laws that the agent 
has in her memory is restricted, and so is the set of logical consequences of all what she knows explicitly. In this way we can achieve a good tradeoff between logical omniscience and logical ignorance: the agent is surely not omniscient with respect to her actual or explicit knowledge, but neither is she logically ignorant. Our task is to find a suitable way to express this idea formally.

\section{Dynamizing Epistemic Logic}

Let us consider an inference rule, say $R$. It can be a valid inference rule of classical logic, or some other (non-classical) logic, for example, intuitionist logic, conditional logic or relevant logic. Assume that an agent accepts $R$ as valid and she can use $R$. What does it mean? In the modal approach we formalize this idea by an axiom saying that the knowledge set of the agent is closed under this rule, that is, if all premises of the rule are known then the conclusion of $R$ is also known. However, as we noted above, it is only true of implicit knowledge. In the context of explicit knowledge it must mean something different. It means rather that, if the agent knows all premises of the rule, and if she perform the inference according to the rule $R$, then she will know the conclusion. The agent does not know the conclusion automatically, but rather as the result of some action, viz. the (mental) action of performing the corresponding inference. If she does not perform this action, then we cannot require her to know the conclusion, although this conclusion may seem to be an obvious consequences of the sentences under consideration. Especially, a logical axiom can be viewed as an inference rule without any premises. We cannot require the agent to know all axioms automatically and permanently, she must rather carry out some action before she can acquire knowledge of a certain axiom. It is possible that the agent knows all logical truths, but merely in principle. This knowledge is only implicit. Factually she never knows them all at once explicitly.

For formalizing the reasoning actions it is natural to use (a form of) dynamic logic (cf. [12], [8].) Thus, we can add a set of basic actions to the language of epistemic logic. The set of formulae now includes formulae like $\left[R_{i}\right] K_{i} A$ or $\left\langle R_{i}\right\rangle K_{i} A$ with the intended meaning: "always after using rule $R$ (or sometimes after using $R$ ) the agent $i$ knows $A$ ". The formalization of the idea that an agent accepts and is able to use an inference rule is straightforward. For example, the idea that the agent $i$ accepts modus ponens can be formalized by the axiom: $K_{i} A \wedge K_{i}(A \rightarrow B) \rightarrow\left\langle M P_{i}\right\rangle K_{i} B$. This axiom says no more than if agent $i$ knows $A$ and she also knows that $A$ implies $B$, then after a suitable inference step she will know $B .^{2}$

As the axioms can be viewed as special inference rules we can introduce an action corresponding to each agent and each axiom of the basis logic, which describes the ability of the agent to use this axiom in her reasoning. (In general, different agents may have different logics, so that the sets of basic actions are different for different agents. However, in the paper we assume a set of homogeneous agents, for the sake of simplicity.) By means of the familiar program connectives for dynamic logic (such as composition or iteration) we can formalize the idea that the agent may know the

\footnotetext{
${ }^{2}$ Instead of $K_{i} A \rightarrow\left\langle R_{i}\right\rangle K_{i} B$ we could also introduce a binary operator $K_{i} A\left\langle R_{i}\right\rangle K_{i} B$ with the interpretation "in a state where the agent $i$ knows $A$, after the application of the rule $R$ she may know $B$ ". However, the former notation is closer to that of dynamic logic, whereas the latter one does not offer any obvious advantage.
} 
consequences of some sentence which she already knows explicitly, provided that she performs the right reasoning steps. For example, assume that the agent $i$ knows the conjunction of $A$ and $A \rightarrow B$, that is, $K_{i}(A \wedge(A \rightarrow B))$. In all normal modal systems we can deduce $K_{i}(A \wedge B)$. However, this inference is not sound for realistic agents. There is no guarantee that the agent will know $A \wedge B$ automatically, as the modal approach suggests. We can only say that if the agent reasons correctly, then she will know $A \wedge B$. In our concrete case, let CE, CI, MP be the conjunction elimination rule, the conjunction introduction rule, and modus ponens, respectively, and let the symbol ";" denote the composition of actions. We write $(C E ; M P ; C I)_{i}$ as an abbreviation for $C E_{i} ; M P_{i} ; C I_{i}$ ("agent $i$ performs $C E$, then $M P$, and then $C I$ ".) Then our theorem must be: $K_{i}(A \wedge(A \rightarrow B)) \rightarrow\left\langle(C E ; M P ; C I)_{i}\right\rangle K_{i}(A \wedge B)$, and not $K_{i}(A \wedge(A \rightarrow B)) \rightarrow K_{i}(A \wedge B)$ as in the standard modal approach.

In general, suppose that $B$ follows from $A$ in some basis logic (which is accepted by the agent) and that the agent knows $A$. For explicit knowledge we cannot assume that the agent automatically knows $B$. Let a proof of $B$ from $A$ be given, where the axioms and inference rules used in the proof are $R_{1}, \ldots, R_{n}$ (in this order, where the same axiom or inference rule may occur at different places in the sequence.) Then, instead of the monotonicity rule in the standard modal approach we have the axiom: $K_{i} A \rightarrow\left\langle\left(R_{1} ; \ldots ; R_{n}\right)_{i}\right\rangle K_{i} B$. This axiom says that if the agent $i$ performs the sequence of actions corresponding to the rules from $R_{1}$ to $R_{n}$ (in this order) then she may know $B$ under the given circumstances. Whether or not the agent can come to this conclusion depends crucially on her logical ability. In this way we see that the logical omniscience problem can be solved easily in a natural way: we can describe agents whose knowledge may or may not be closed under logical laws. On the other hand we can still say that the agent thinks rationally, that she is not logically ignorant. Theoretically she may produce all logical truths, and all logical consequences of her knowledge, but only if she is interested in doing so, if she has enough time and memory, et cetera.

In the above argumentation we have made an implicit assumption. We have assumed that all premises, once known by the agent, are still available after the agent performs a reasoning step. In the previous example, if the agent forgets the premise $A$ immediately after using modus ponens, then she cannot apply the conjunction introduction rule to come to the conclusion $A \wedge B$. Thus, we have to postulate that the agent does not forget what she previously knows after performing some reasoning action. This assumption can be formalized using persistence axioms for knowledge, for example, $K_{i} A \rightarrow\left[R_{i}\right] K_{i} A$.

Are such persistence axioms reasonable? Only under two conditions. First, the truth value of $A$ should not change over time. If $A$ becomes false after $i$ 's inference using rule $R$ then it is not reasonable to postulate that $i$ still knows $A$ after the use of $R$. This point should be taken into account when we formally define the language of our logic. In particular, if our language contains temporal indexicals then sentences containing them cannot be regarded as persistent. Second, the truth value of $A$ may not change through the agent's actions. This excludes formulae such that $\neg K_{i} B$ : it is possible that agent $i$ does not know $B$ now, but will know it as a result of her reasoning. In general, a formula in which a knowledge operator occurs essentially negative (i.e., within the scope of an odd number of the negation sign) is not a suitable candidate for a persistent one. So, we may assume that persistent formulae are built 
up from objective formulae, conjunction, disjunction, and the knowledge operators only.

Let us now examine how the ability of the agents to introspect their knowledge can be captured within our dynamic framework. Let $I_{i}$ be $i$ 's action of introspection. ${ }^{3}$ Consider positive introspection first. Suppose that $i$ knows $A$. Can we infer that she will know after introspecting her knowledge that she knows $A$ ? Not necessarily! We can assume that $i$ will know that she previously knows $A$, but to support the inference that after her introspection action the agent knows that she knows $A$ we need one more argument, namely that $i$ 's knowledge of $A$ will not be changed through her reasoning actions. We have argued previously that such a persistence axiom is reasonable for a subclass of formulae. Thus, we have the following axiom of positive introspection, which corresponds to the schema 4 in modal epistemic logic: $K_{i} A \rightarrow\left\langle I_{i}\right\rangle K_{i} K_{i} A$, provided that $A$ is persistent.

The same argumentation can be used to show that the candidate for the negative introspection axiom $\neg K_{i} A \rightarrow\left\langle I_{i}\right\rangle K_{i} \neg K_{i} A$ is not acceptable. It can happen that after a reasoning step the agent knows something what she did not know previously. If we extend the language to include objective or absolute time, then a statement such as $\neg K_{i}^{t} A \rightarrow\left\langle I_{i}\right\rangle K_{i}^{t+1} \neg K_{i}^{t} A$ would be absolutely reasonable. However, this issue will not be pursued further in the present paper.

In order to define systems of dynamic epistemic logic formally we can fix a basis logic and then associate with each axiom schema and each inference rule an atomic action. The "external" language (i.e., the language in which one can speak about agents) is then defined over this set of atomic actions. The "external" logic comprises all theorems of dynamic logic and the specific epistemic axioms discussed above.

However, there are some problems with this approach. First, there might be many different, but equivalent axiomatizations of the basis logic, so the choice of the basic actions must be arbitrary. Moreover, as the resulting dynamic-epistemic system contains dynamic logic entirely, it becomes very complex and therefore unhandly. Even more importantly, in most cases we do not need to care about what course of actions the agents just carried out; we are only interested in the result of the actions, so to speak. We only need to know that a certain agent has carried out some reasoning steps, and after that she gains certain new information.

This last point leads us to another approach. We introduce an auxiliary action $F_{i}$ with the following intended reading: do any one of the atomic actions (we don't know what action;) repeat the non-deterministic choice finitely many times (at least once, but we don't know how many times!) The action $F_{i}$ could be interpreted as a course of thought of the agent $i$. From the viewpoint of dynamic logic: if the set of all atomic actions associated with the agent $i$ and her basis logic is a finite set $\left\{r_{i}^{1}, \ldots, r_{i}^{n}\right\}$, then $F_{i}$ can be viewed as $\left(r_{i}^{1} \cup r_{i}^{2} \cup \ldots \cup r_{i}^{n}\right)^{+}$, where the symbols $\cup$ and ${ }^{+}$denote choice and non-zero iteration, respectively. ${ }^{4}$ The choice of the symbols $F_{i}$ is not accidental at all: in temporal logic it stands for the operator "Future". It turns out that our

\footnotetext{
${ }^{3}$ One may ask how seriously one can take introspection as action. Well, it is true that introspection may differ from the "genuine" reasoning actions in some aspects. However, the differences are not quite significant. It seems reasonable to treat introspection as test of a certain kind, which is used by the agents to reason about their own mental state.

${ }^{4}$ In dynamic logic another form of iteration is considered, viz. the one that allows for running a program zero time, denoted by *. But one can easily extend dynamic logic to include non-zero iteration as well.
} 
auxiliary action behaves in the same manner as the future operator of temporal logic: the operator $\langle F\rangle$ satisfies all the axioms for the minimal temporal logic $\mathrm{K}_{\mathrm{t}} 4$. It is no surprise at all: we know that the minimal temporal logic can be embedded into dynamic logic, and one way to do this is to take the iteration of an action to interpret the future operator. The formal language in which our dynamic epistemic logics are formulated is called $L_{D E}$ and will be defined in the following section.

\section{Dynamic Epistemic Logic}

\subsection{The language of dynamic epistemic logic}

Definition 3 (The language $L_{D E}$ ) Let $A g t=\{1, \ldots, n\}$ be a set of $n$ agents and let $L_{E}$ be the language of epistemic logic as defined in Definition 1. $L_{D E}$ is the least set such that

1. $L_{E} \subseteq L_{D E}$

2. If $A \in L_{D E}$ then $\neg A \in L_{D E}$

3. If $A \in L_{D E}$ and $B \in L_{D E}$ then $(A \rightarrow B) \in L_{D E}$

4. If $A \in L_{D E}$ then $\left\langle F_{i}\right\rangle A \in L_{D E}$

Conjunction, disjunction and the operator $\left[F_{i}\right]$ dual to $\left\langle F_{i}\right\rangle$ are defined as usual. The formula $\left\langle F_{i}\right\rangle A$ is read: " $A$ is true after some course of thought of $i$ ", $\left[F_{i}\right] A$ means " $A$ is true after any course of thought of $i$ ". (We could think of $\left\langle F_{i}\right\rangle$ and $\left[F_{i}\right]$ as the modalities "at some future times" and "at all future times" of temporal logic, but now time is subjective time, i.e., agent-dependent, generated by the agent's actions.) Note that we do not allow the operator $\left\langle F_{i}\right\rangle$ to occur inside the scope of any knowledge operator. The reason is that such expressions are indexicals: they contain temporal indexicals like "later" or "always" implicitly. We want to exclude indexical expressions from our language because they require special treatment, which could be very involved and may obscure more important points.

Definition 4 The sublanguage $L_{E}^{+}$of $L_{E}$ is the smallest set of formulae from $L_{E}$ which contains all objective formulae and is closed under the condition: if $A, B \in L_{E}^{+}$ and $i \in A g t$ then $\left\{(A \wedge B),(A \vee B), K_{i} A\right\} \subseteq L_{E}^{+}$.

\subsection{The system DES4 $4_{n}$}

Now we go on to define an axiomatic system for reasoning about the dynamics of knowledge along the lines described in the previous section. We have three groups of axioms: the usual axioms of the propositional calculus, axioms for temporal logic, and axioms governing the interaction between knowledge and reasoning activities.

Definition 5 (The system DES4n ) The logic DES4 $4_{n}$ (Dynamic-Epistemic $4_{n}$ ) has the following axiom schemata:

PC1. $A \rightarrow(B \rightarrow A)$ 
PC2. $(A \rightarrow(B \rightarrow C)) \rightarrow((A \rightarrow B) \rightarrow(A \rightarrow C))$

PC3. $(\neg B \rightarrow \neg A) \rightarrow(A \rightarrow B)$

TL1. $\left[F_{i}\right](A \rightarrow B) \rightarrow\left(\left[F_{i}\right] A \rightarrow\left[F_{i}\right] B\right)$

TL2. $\left[F_{i}\right] A \rightarrow\left[F_{i}\right]\left[F_{i}\right] A$

DE1. $K_{i} A \wedge K_{i}(A \rightarrow B) \rightarrow\left\langle F_{i}\right\rangle K_{i} B$

DE2. $K_{i} A \rightarrow A$

DE3. $K_{i} A \rightarrow\left[F_{i}\right] K_{i} A$, provided that $A \in L_{E}^{+}$

DE4. $\left\langle F_{i}\right\rangle K_{i}(A \rightarrow(B \rightarrow A))$

DE5. $\left\langle F_{i}\right\rangle K_{i}((A \rightarrow(B \rightarrow C)) \rightarrow((A \rightarrow B) \rightarrow(A \rightarrow C)))$

DE6. $\left\langle F_{i}\right\rangle K_{i}((\neg B \rightarrow \neg A) \rightarrow(A \rightarrow B))$

DE7. $\left\langle F_{i}\right\rangle K_{i}\left(K_{i} A \rightarrow A\right)$

DE8. $K_{i} A \rightarrow\left\langle F_{i}\right\rangle K_{i} K_{i} A$, provided that $A \in L_{E}^{+}$

The rules of inference are:

R1. Modus ponens: if $A$ and $A \rightarrow B$ are theorems then $B$ is a theorem.

R2. Necessitation: if $A$ is a theorem then so is $\left[F_{i}\right] A$.

In these axioms and rules, the index $i$ ranges over the whole set $A g t$ of agents. The axioms PC1 - PC3 together with the rule R1 axiomatize completely the propositional calculus. Together with TL1, TL2 and R2 they form a complete axiomatization of the minimal temporal logic of transitive time. The axioms DE1 - DE7 describe the dynamics of knowledge. Axiom DE1 says that the agents are capable of using modus ponens. Axiom DE2 is the well-known schema T saying that knowledge entails truth. Axiom DE3 says that agents do not forget what they know when they are reasoning. Axioms DE4 - DE6 state that the agents are able to use the axioms PC1 - PC3 of classical logic in their reasoning. Axiom DE7 says that agents potentially trust their knowledge: when thinking about themselves, they think that what they know must be true (as opposed to what they merely believe.) Finally, DE8 says that the agents are capable of positive introspection. Of course, only instances of these schemata which are well-formed formulae are allowed.

The notions of a proof, a theorem, and a consistent formula or set of formulae (with respect to the logic DES4 $4_{n}$ ) are defined as usual. The provability relation is denoted $\vdash_{\mathrm{DES} 4_{\mathrm{n}}}$, where the index may be omitted if no confusion can occur. Moreover, we say that a formula $A \in L_{E}$ is PC-provable, in symbol $\vdash_{P C} A$, just in case $A$ can be proved using only instances of the schemata PC1 - PC3 (in the sublanguage $L_{E}$ ) and modus ponens.

Of course, we can postulate that the agents can use further simple tautologies and inference rule in their reasoning. For example, we can include axioms such that $K_{i} A \wedge K_{i} B \rightarrow\left\langle F_{i}\right\rangle K_{i}(A \wedge B)$, or $\left\langle F_{i}\right\rangle K_{i}(A \vee \neg A)$. However, this is not necessary at all, because they can be proved, as we shall see later. 


\subsection{Some features of DES4}

Theorem 6 (Consistency) The system DES4 $4_{n}$ is consistent.

Proof To see that the system DES4 $4_{n}$ is consistent, i.e., no contradiction can be derived from it, it suffices to notice that all axioms and inference rules of DES4 $4_{n}$ can be mapped to valid formulae and inference rules of the propositional calculus by deleting all occurrences of $K_{i}$ and $\left\langle F_{i}\right\rangle$ from them. Therefore, all theorems of DES4 $_{n}$ must become propositional tautologies when all occurrences of $K_{i}$ and $\left\langle F_{i}\right\rangle$ are deleted. Hence, a formula like $A \wedge \neg A$ cannot be derived.

The following theorem states that DES4 $4_{n}$ solves the logical omniscience problem. It says that none of the rules NEC, MON, and CGR is valid. Moreover, an agent's explicit knowledge (or her information state) at a time, i.e., the totality of all what this agent knows at that time, needs not be closed under any nontrivial logical rule. ${ }^{5}$

Theorem 7 (Non-Omniscience) 1. The following inference rules are not derivable in DES4 $4_{n}$.

NEC. If $A$ is a theorem then so is $K_{i} A$

MON. If $A \rightarrow B$ is a theorem then so is $K_{i} A \rightarrow K_{i} B$

CGR. If $A \leftrightarrow B$ is a theorem then so is $K_{i} A \leftrightarrow K_{i} B$

2. The following formulae are not provable:

(a) $K_{i}(A \rightarrow B) \rightarrow\left(K_{i} A \rightarrow K_{i} B\right)$

(b) $K_{i} B \rightarrow K_{i}(A \rightarrow B)$

(c) $K_{i}(A \wedge B) \rightarrow K_{i} A$

(d) $K_{i}(A \wedge B) \rightarrow K_{i} A \wedge K_{i} B$

(e) $K_{i} A \wedge K_{i} B \rightarrow K_{i}(A \wedge B)$

(f) $K_{i} A \rightarrow K_{i}(A \vee B)$

(g) $K_{i} A \vee K_{i} B \rightarrow K_{i}(A \vee B)$

(h) $K_{i} \neg \neg A \rightarrow K_{i} A$

(i) $K_{i} A \rightarrow K_{i} K_{i} A$

(j) $\neg K_{i} A \rightarrow K_{i} \neg K_{i} A$

Proof We can construct easily interpretations such that (i) all axioms of DES4 ${ }_{n}$ are valid, (ii) the DES4 $4_{n}$-rules of inference lead from valid formulae to valid ones, and (iii) the formulae and rules listed above are invalidated. We omit the details.

\footnotetext{
${ }^{5}$ An agent's knowledge is of course closed under trivial rules like $A \vdash A$ : a formula like $K_{i} A \rightarrow K_{i} A$ should be in any case valid. But such a formula does not say anything about the agent's reasoning capacities. It is not a genuine epistemic statement.
} 
An agent described by the logic DES4 $\mathrm{n}$ is not logically omniscient. On the other hand, we cannot say that she is not rational: the agent is rational, because she can (at least in principle) perform actions to close her knowledge under logical laws, as the following theorems show. Instead of the necessitation rule and monotony rule in modal epistemic logic we have now a theorem stating that the agents can know all classical theorems and can draw all consequences of what they know, provided that they perform the right reasoning.

Theorem 8 Let $A, B$ be objective formulae. The following inference rules are derivable in $\mathrm{DES}_{\mathrm{n}}$ :

R3. If $\vdash_{P C} A$ then $\vdash_{\mathrm{DES}_{4}}\left\langle F_{i}\right\rangle K_{i} A$.

R4. If $\vdash_{P C} A \rightarrow B$ then $\vdash_{\mathrm{DES}_{4}} K_{i} A \rightarrow\left\langle F_{i}\right\rangle K_{i} B$.

Proof See the appendix.

Corollary 9 Assume that $A, B$ are objective formulae. The following formulae are theorems of DES4n:

1. $K_{i} B \rightarrow\left\langle F_{i}\right\rangle K_{i}(A \rightarrow B)$

2. $K_{i}(A \wedge B) \rightarrow\left\langle F_{i}\right\rangle K_{i} A$

3. $K_{i}(A \wedge B) \rightarrow\left\langle F_{i}\right\rangle K_{i} A \wedge\left\langle F_{i}\right\rangle K_{i} B$

4. $K_{i} A \rightarrow\left\langle F_{i}\right\rangle K_{i}(A \vee B)$

5. $K_{i} A \vee K_{i} B \rightarrow\left\langle F_{i}\right\rangle K_{i}(A \vee B)$

6. $K_{i} \neg \neg A \rightarrow\left\langle F_{i}\right\rangle K_{i} A$

In fact, the above rules and theorems are derivable for a larger class of formulae, not only for objective ones. The following list comprises some more provable formulae of DES4 $4_{n}$. They say that if all premises of a valid inference rule are known or will be known, then after some steps of reasoning the agents will know the conclusion. We still assume that $A$ and $B$ are objective. Their proofs are found in the appendix.

Theorem 10 The following formulae are theorems of DES4 $4_{n}$ :

1. $K_{i} A \wedge\left\langle F_{i}\right\rangle K_{i}(A \rightarrow B) \rightarrow\left\langle F_{i}\right\rangle K_{i} B$

2. $K_{i} A \wedge K_{i} B \rightarrow\left\langle F_{i}\right\rangle K_{i}(A \wedge B)$

3. $K_{i} A \wedge\left\langle F_{i}\right\rangle K_{i} B \rightarrow\left\langle F_{i}\right\rangle K_{i}(A \wedge B)$

4. $K_{i}(A \wedge B) \rightarrow\left\langle F_{i}\right\rangle\left(K_{i} A \wedge K_{i} B\right)$ 


\section{Conclusions and Open Problems}

\subsection{Summary}

We have shown how to solve the logical omniscience problem of epistemic logic while preserving the intuition that the agents are logical, rational beings. Our strategy consists in taking the dynamic aspect of knowledge into account. We have argued that the correct form of an axiom for epistemic should be: if an agent knows all premises of a valid inference rule, and if she performs the right reasoning, then she will know the conclusion as well. Our strategy can do justice to the intuition that the agents are neither logically omniscient nor logically ignorant. They are non-omniscient, because their actual (or explicit) knowledge at a single time point needs not be closed under any logical law. It is even possible that they do not know any logical truth at all at some of their information states. On the other hand, they are non-ignorant, because they are capable of logical thinking. They can use their reasoning capacities to infer new information from what they already know. Their rationality is not restricted by any artificial, ad hoc postulate saying that their inference mechanisms are incomplete. If an agent performs the correct inferences and if she has enough time, then she might arrive at an ideal information state where all logical consequences of her current beliefs have been drawn. This ideal state can never be achieved by real agents, but this is another matter.

\subsection{Related works}

To our knowledge, there exists no similar work in the literature which pursues the strategy of dynamizing epistemic logic in order to solve the dilemma of logical omniscience and logical ignorance. Most close to our approach are perhaps works on "parameterized epistemic logic", proposed e.g. by Stelzner ([23],) where knowledge is time (agent, context ...) dependent. However, in his formal systems Stelzner does not consider the concepts of knowledge and belief, but a related concept, the concept of a (hypothetical) obligation to defend some sentence. The latter concept is related to the former in the following way: in a rational discourse, if an agent asserts some sentence, then she has the obligation to defend it when it is challenged, because she has made public through her assertion that she believes in the sentence. Stelzner investigates axioms to describe agents in a rational discourse. These axioms say, for example, that if an agent is obligated to defend $A$ at $t$ and $B$ can be inferred from $A$ by one inference step, then the agent can be obligated to defend $B$ at time $t+1$. (A time line isomorph to the natural numbers, generated by the consecutive "moves" in the discourse, is assumed. The obligation to defend $B$ is only hypothetical, because it does not arise if $B$ is not challenged.) With the aid of such axioms one can classify agents according to their rationality. Stelzner's logic could be reinterpreted as formalizing the concept of implicit, or possible knowledge, but not the concept of explicit, or actual knowledge: a statement such as $K_{i}^{t}(A \wedge B) \rightarrow K_{i}^{t+1} A$ is perhaps more acceptable than the axiom $K_{i}^{t}(A \wedge B) \rightarrow K_{i}^{t}(A)$, but it is still a too strong requirements for the notion of actual knowledge. In contrast, We have tried to show how the concept of actual knowledge can be captured.

In the literature on belief revision some authors have considered belief-changing actions. For example, Van Linder, van der Hoek and Meyer ([19], [20]) have done 
some work to formalize the change of knowledge through actions. However, they made very strong assumptions about knowledge: their agents are logically omniscient. The actions they consider lead from one deductively closed belief set to another. Thus, their work should be read in terms of information dynamics, and not knowledge dynamics.

\subsection{Future directions}

We can develop variants of $\mathrm{DES}_{\mathrm{n}}$ to describe different sorts of agents. For instance, we could modify the axiom system to formalize the concept of belief. We can base epistemic logic on another, non-classical logic. We could also add some more axioms or drop some of the axioms of DES4n. How to do it concretely depends crucially on our intended application. At the moment we are working to integrate our dynamic epistemic logic into a framework for reasoning about actions in multi-agent systems.

By defining the proof system DES4 ${ }_{n}$ we have provided a procedural semantics for the concept of knowledge. It remains unclear whether or not the system DES4 comprises all valid formulae of the language $L_{D E}$. To answer this question we need to develop an intuitively acceptable declarative semantics for the concept of knowledge. It is possible to develop a model theory along the lines of [4], so that we may prove completeness of DES4 $4_{n}$ with respect to the defined models. However, such a model theory is simply a reformulation of the procedural semantics, it does not provide us with a tool to determine if all valid epistemic statements have been captured by the proof system. A well motivated and intuitively acceptable semantics should allow us to analyze the epistemic concepts of knowledge and belief in term of simpler and more fundamental concepts. The appeal to the concepts of "epistemic alternatives" and "possible worlds" does not help, as the problems of the modal approach show.

Another open issue is to find a way to incorporate indexical knowledge in our framework. We have so far ignored this issue and exclude indexical expressions from our language. However, for many applications we must be able to treat indexical knowledge adequately. Some work has been done on this issue, e.g. by Lespérance and Levesque ([17].) However, their and related works should be seen as dealing with indexical information (or indexical possible knowledge), and not with genuine indexical knowledge, for the reasons explained earlier. Thus, much work still remains to be done.

So far our logic has been monotonic in two aspects. First, the consequence operation of DES4 $\mathrm{n}$ is monotonic. Second, the knowledge of the agents always grows over time. A very interesting, still open problem is to develop dynamic epistemic logics based on non-monotonic logic, where the agents can revise their knowledge when they find out that their knowledge is inconsistent. We may expect to find interesting connections with two other, very active fields of AI research, viz. to non-monotonic reasoning and to the logic of belief revision. This seems to be a promising field of research and needs further investigations.

\section{Acknowledgments}

I thank my teachers Peter Steinacker and Heinrich Herre for their invaluable help during the last several years. My view on epistemic logic is strongly influenced by 
Werner Stelzner's works. I thank the anonymous referees for their helpful comments. I had the chance to discuss preliminary versions of this paper with several people on different occasions. I thank all of them for their comments which have helped me very much to improve the presentation of the paper.

\section{References}

[1] J. Barwise. Three views of common knowledge. In The Situation in Logic. CSLI, Stanford, 1989.

[2] B. Chellas. Modal Logic. An Introduction. Cambridge UP, Cambridge, 1980.

[3] H. N. Duc. Ein system der epistemischen logik. In W. Stelzner, editor, Philosophie und Logik. Frege-Kolloquien Jena 1989/1991, pages 205-214, Berlin and New York, 1993. Walter de Gruyter.

[4] H. N. Duc. Logical omniscience vs. logical ignorance. On a dilemma of epistemic logic. In C. P. Pereira and N. Mamede, editors, Progress in Artificial Intelligence. Proceedings of EPIA'95, volume 990 of LNAI, pages 237-248, Heidelberg, 1995. Springer Verlag.

[5] R. Eberle. A logic of believing, knowing and inferring. Synthese, 26:356-382, 1974.

[6] R. Fagin and J. Y. Halpern. Belief, awareness and limited reasoning. Artificial Intelligence, 34:39-76, 1988.

[7] R. Fagin, J. Y. Halpern, Y. Moses, and M. Y. Vardi. Reasoning about Knowledge. MIT Press, 1995.

[8] R. Goldblatt. Logics of Time and Computation. CSLI, Stanford, 1987.

[9] J. Y. Halpern. Reasoning about knowledge: An overview. In J. Y. Halpern, editor, Proceedings of the 1986 Conference on Theoretical Aspects of Reasoning About Knowledge, pages 1-18. Morgan Kaufman, 1986.

[10] J. Y. Halpern. Using reasoning about knowledge to analyze distributed systems. Annual Review of Computer Science, 2:37-68, 1987.

[11] J. Y. Halpern and Y. Moses. A guide to completeness and complexity for modal logics of knowledge and belief. Artificial Intelligence, 54:319-379, 1992.

[12] D. Harel. Dynamic logic. In D. Gabbay and F. Guenthner, editors, Handbook of Philosophical Logic, volume II, pages 497-604. Reidel, Dordrecht, 1984.

[13] J. Hintikka. Knowledge and Belief. Cornell UP, Ithaca, New York, 1962.

[14] J. Hintikka. Impossible possible worlds vindicated. Journal of Philosophical Logic, 4:475-484, 1975.

[15] M. Hocutt. Is epistemic logic possible? Notre Dame Journal of Formal Logic, 13:433-453, 1972. 
[16] K. Konolige. A Deduction Model of Belief. Pitman Publishing, London, 1986.

[17] Y. Lespérance and H. Levesque. Indexical knowledge and robot action-a logical account. Artificial Intelligence, 73:69-115, 1995.

[18] H. Levesque. A logic of implicit and explicit belief. In Proceedings AAAI-84, pages 198-202, Austin, TX, 1984.

[19] B. van Linder, W. van der Hoek, and J.-J. Ch. Meyer. Actions that make you change your mind: Belief revision in an agent-oriented setting. Technical Report UU-CS-1994-53, Utrecht University, December 1994.

[20] B. van Linder, W. van der Hoek, and J.-J. Ch. Meyer. The dynamics of default reasoning (extended abstract). In C. Froidevaux and J. Kohlas, editors, Symbolic and Quantitative Approaches to Reasoning and Uncertainty (Proceedings of ECSQARU'95), volume 946 of Lecture Notes in Computer Science (subseries LNAI), pages 277-284. Springer-Verlag, 1995.

[21] V. Rantala. Impossible worlds semantics and logical omniscience. Acta Philosophica Fennica, 35:18-24, 1982.

[22] P. Steinacker. Superschwache Modalkalküle und einige epistemische Anwendungen. PhD thesis, Universität Leipzig, 1979.

[23] W. Stelzner. Epistemische Logik. Akademie-Verlag, Berlin, 1984.

[24] W. Stelzner. Epistemische und deontische logik. In L. Kreiser, S. Gottwald, and W. Stelzner, editors, Nichtklassische Logik, pages 181-224. Akademie-Verlag, Berlin, 1988.

[25] K. Wuttich. Glauben, Zweifel, Wissen. Eine logisch-philosophische Studie. Akademie-Verlag, Berlin, 1991.

\section{A Formal Proofs}

\section{A.1 Proof of Theorem 8}

First, note that $\left[F_{i}\right] A \wedge\left\langle F_{i}\right\rangle B \rightarrow\left\langle F_{i}\right\rangle(A \wedge B)$ and $\left\langle F_{i}\right\rangle\left\langle F_{i}\right\rangle A \rightarrow\left\langle F_{i}\right\rangle A$ are DES4 $4^{-}$ provable. Moreover, if $A \rightarrow B$ is DES4 $4_{n}$-theorem then so is $\left\langle F_{i}\right\rangle A \rightarrow\left\langle F_{i}\right\rangle B$. We shall make extensive use of these facts in our proof without mentioning them explicitly. To shorten the proofs we assume that all theorems and rules of $\mathrm{PC}$ and $\mathrm{K}_{\mathrm{t}} 4$ have been derived, so we do not have to write them down explicitly.

Consider rule R3. Let $\vdash_{P C} A$. We show $\vdash_{\mathrm{DES} 4_{\mathrm{n}}}\left\langle F_{i}\right\rangle K_{i} A$ by induction on the length $m$ of the proof of $A$. If $m=1$ then $A$ must be an instance of one of the axiom schemata PC1-PC3. The claim follows from DE4-DE6. If $m>1$ then $A$ must be obtained by applying modus ponens from, say, $B$ and $B \rightarrow A$, which are PC-provable in less than $m$ steps. So we assume that there is a PC-proof of $A$ of length $m$ where in the $k$-th and $l$-th lines we had proved $B$ and $B \rightarrow A$. The PC-proof of $A$ can be extended to a DES4 $4_{\mathrm{n}}$-proof of $K_{i} A$ as follows: 


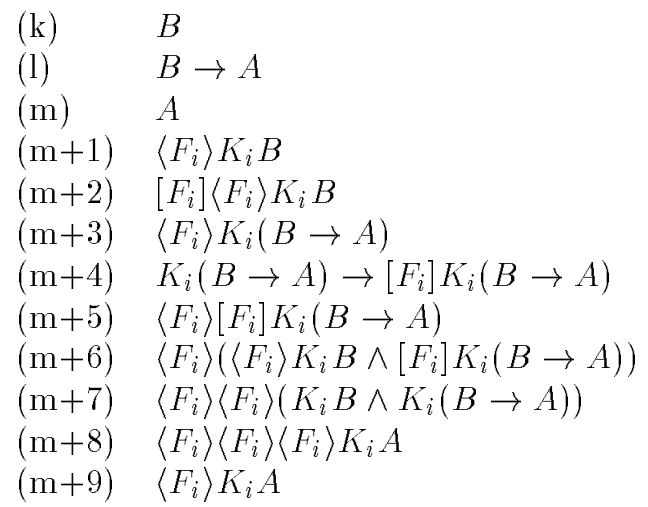

Ass.

Ass.

(k), (l), R1

Ind. Hyp., (k)

$(\mathrm{m}+1), \mathrm{R} 2$

Ind. Hyp., (l)

DE3

$(\mathrm{m}+3),(\mathrm{m}+4)$

$(m+2),(m+5)$

$(\mathrm{m}+6)$

$(\mathrm{m}+7), \mathrm{DE} 1$

$(m+8)$

Ass.

Ass.

(1), R3

DE3, (2)

(3), (4)

(5), DE1

(6)

(2), (7)

\section{A.2 Proof of Theorem 10}

1. $K_{i} A \wedge\left\langle F_{i}\right\rangle K_{i}(A \rightarrow B) \rightarrow\left\langle F_{i}\right\rangle K_{i} B$

$\begin{array}{ll}(1) & K_{i} A \\ (2) & \left\langle F_{i}\right\rangle K_{i}(A \rightarrow B) \\ (3) & {\left[F_{i}\right] K_{i} A} \\ (4) & \left\langle F_{i}\right\rangle\left(K_{i}(A \rightarrow B) \wedge K_{i} A\right) \\ (5) & \left\langle F_{i}\right\rangle\left\langle F_{i}\right\rangle K_{i} B \\ (6) & \left\langle F_{i}\right\rangle K_{i} B\end{array}$

Ass.

Ass.

DE3, (1)

(2), (3)

(4), DE1

(5)

2. $K_{i} A \wedge K_{i} B \rightarrow\left\langle F_{i}\right\rangle K_{i}(A \wedge B)$
(1) $K_{i} A$
(2) $\quad K_{i} B$
(3) $\quad\left[F_{i}\right] K_{i} A$
(4) $\quad\left[F_{i}\right] K_{i} B$
(5) $\quad\left\langle F_{i}\right\rangle K_{i}(A \rightarrow(B \rightarrow(A \wedge B)))$
$\left\langle F_{i}\right\rangle\left(K_{i} A \wedge K_{i}(A \rightarrow(B \rightarrow(A \wedge B)))\right)$
$\left\langle F_{i}\right\rangle K_{i}(B \rightarrow(A \wedge B))$
$\left\langle F_{i}\right\rangle\left(K_{i} B \wedge K_{i}(B \rightarrow(A \wedge B))\right)$
$\begin{array}{ll}(8) & \left\langle F_{i}\right\rangle\left(K_{i} B \wedge K_{i}\right. \\ (9) & \left\langle F_{i}\right\rangle K_{i}(A \wedge B)\end{array}$
Ass.
Ass.
DE3, (1)
DE3, (2)
R3
(3), (5)
(6), DE1
(4), (7)
(8)

3. $K_{i} A \wedge\left\langle F_{i}\right\rangle K_{i} B \rightarrow\left\langle F_{i}\right\rangle K_{i}(A \wedge B)$ 

(1) $\quad K_{i} A$
(2) $\left\langle F_{i}\right\rangle K_{i} B$
(3) $\quad\left[F_{i}\right] K_{i} A$
(4) $\left\langle F_{i}\right\rangle\left(K_{i} A \wedge K_{i} B\right)$

4. $K_{i}(A \wedge B) \rightarrow\left\langle F_{i}\right\rangle\left(K_{i} A \wedge K_{i} B\right)$
(1) $\quad K_{i}(A \wedge B)$
(2) $\left\langle F_{i}\right\rangle K_{i} A$
(3) $\left\langle F_{i}\right\rangle\left[F_{i}\right] K_{i} A$
(4) $\quad\left[F_{i}\right] K_{i}(A \wedge B)$
(5) $\left[F_{i}\right]\left\langle F_{i}\right\rangle K_{i} B$
(6) $\quad\left\langle F_{i}\right\rangle\left(\left[F_{i}\right] K_{i} A \wedge\left\langle F_{i}\right\rangle K_{i} B\right)$
(7) $\quad\left\langle F_{i}\right\rangle\left\langle F_{i}\right\rangle\left(K_{i} A \wedge K_{i} B\right)$
(8) $\quad\left\langle F_{i}\right\rangle\left(K_{i} A \wedge K_{i} B\right)$

Ass.

Ass.

DE3, (1)

(3), (2)

Ass.

(1), R4

(2), DE3

(1), DE3

(4), R4

(3), (5)

(6)

(7) 